\title{
Social Media and the Black Travel Community: From Autonomous Space to Liberated Space
}

\author{
Tonia Sutherland \\ University of Hawai'i at Mānoa \\ tsuther@hawaii.edu
}

\begin{abstract}
This paper reports on findings from an ongoing study of identity-based social media communities that subvert the architecture of internet and other digital tools to evolve autonomous ("safe") spaces into liberated spaces. The community in question endeavors to provide safe spaces for information and resource sharing. Two compelling trends were found. The first involves entrepreneurship as a feature of liberated spaces. The second involves secret, or "underground" communication hidden in open spaces as a liberatory practice. As part of discussing these trends, the author introduces the idea of liberated spaces and argues for their importance within discussions of the sociocultural aspects of inclusive digital interfaces and digital cultures.
\end{abstract}

\section{Introduction}

Online social media communities offer members the opportunity to establish and join spaces populated by similar people, or people with similar interests. Membership in social media communities is voluntary and is often subject to approval by a community administrator. Some communities are invitation-only: membership requires invitation by a current member or the community administrator. As autonomous spaces, social media communities also vary by online visibility: some are public, while others are private or secret and only visible to members. Because of these affordances, it has been common for social media communities to be designed as autonomous, or "safe" spaces, where marginalized people can share their experiences with marginalization and oppression.

Over the past five years, a growing body of scholarship has addressed the unique uses of social media platforms by ethnic minorities and other marginalized populations, examining the ways that these groups use and adapt social media platforms and other digital tools to communicate, form identity and community, and subvert cultures of oppression. Some scholars have addressed the broader racial politics of social media [1, 19], while others have specifically considered the use of social media platforms by marginalized communities, addressing online discourse and cultural performance $[4,5,12$, 13], online racialized identity construction $[14,20]$, racialized violence in online spaces [18, 21], and social media activism and dissent $[2,8,9,15,22]$. This study offers an intervention at the intersection of these concerns, examining how identity-based social media communities subvert the architecture of internet and other digital tools to evolve autonomous spaces into spaces of liberatory potential.

In this paper, I extend the research on autonomous space social media communities to expand understandings of how these safe spaces have also become places of liberation. In conceptualizing the liberated space, I direct my efforts at two distinct categories of social media communities: Facebook Groups and Twitter hashtag communities. Many social media communities have developed as lively spaces where people are able ask frank and challenging questions-without judgment-in psychologically safe environments. Thus, they emerge as "safe" or "autonomous" spaces regardless of the established level of community privacy. Social psychologist Kurt Lewin introduced the idea of safe spaces in his work on group dynamics and organizational change management, which focused in particular, on the problems of minority or disadvantaged groups [16]. Lewin's definition of a "group," applies unambiguously to the social media communities studied here: "... it is not the similarity or dissimilarity of individuals that constitutes a group, but interdependence of fate." Organized around the burgeoning Black Travel Movement, in which community (or "group") trust can be a matter of life and death, members of these social media 
communities rely on the safety of engineered autonomous spaces, while at the same time collaboratively building liberatory spaces within them.

\section{The Black Travel Movement}

Social media communities tend to form around a small number primary themes, including shared common backgrounds; like qualifications; and mutual hobbies and/or interests. Those with a shared common background include assemblages such as family groups, schoolmates (past and present), and those with shared ethnic, religious, or other identity-based traits. Social media communities that form around like qualifications often share similar occupational responsibilities or comparable job titles. Online communities also frequently form around interests and hobbies such as sports, food, travel, social causes, and political affiliations. Frequently there is categorical overlap: a group may be dedicated to people of a given religious affiliation with a particular interest in a social cause, for example. Another example is the growing community of Black Americans interested in travel.

Once confined to occupational travel, the Black Travel Community has grown and evolved significantly since the 1940s. As Black Americans moved from agricultural labor in the South to industrial labor in the North, more people found themselves with disposable income and leisure time for travel. At the same time, the mass production of personal automobiles made it possible for Black Americans to avoid travel on the segregated-and frequently dangerous - railway systems. Finally, the evolution of the American road map from pathfinders to the AAA TripTik made road navigation exponentially easier. The convergence of these developments meant that more Black Americans began to travel for leisure; unfortunately, leisure travel for Black Americans was stymied by oppressive Jim Crow laws that ensured policies of separate-and unequal. Black travelers were frequently denied access to the basic requirements of road travel: some traveled with buckets because public restrooms and roadside rest areas were restricted from use by Black people. They also traveled with extra gasoline and food because they could not use gas stations and were barred from restaurants. As John A. Williams said, it required "nerve and courage" to drive in America if you were Black [23].

To combat the tyranny of Jim Crow, and to offer Black travelers safe passage on American roads, in 1932, a postal worker in Harlem named Victor Hugo Green wrote The Negro Motorists Green Book "to give the Negro traveler information that will keep him from running into difficulties, embarrassments and to make his trip more enjoyable [6]." Colloquially known as The Green Book, Green's book began as a New York publication, eventually expanding to cover all of the United States and beyond in its international edition. Published annually from 1936-1966, The Green Book had advertisements, recommendations, points of interest, and importantly, safety and preparedness tips. The Green Book sold approximately 15,000 copies annually until 1964 when it began to be considered obsolete following the passage of sweeping Civil Rights legislation in the United States. Although Green and The Green Book were criticized during Jim Crow as contributing to segregation rather than fighting for integration, The Green Book did not offer Black travelers the best place to stay or eat (like a modern-day Yelp!) - rather, it drew attention to what was often the only place to stay or eat; particularly in places where the Black population was notably (and intentionally) low-what were commonly known as sundown towns.

Increases in income and education, alongside a tech-savvy generation and the rise of social media have converged to create a contemporary Black Travel Movement (BTM) analogous to the increase in Black travelers in the 1940s and 1950s. Although Victor Hugo Green hoped eventually there would not be a need for The Green Book, Black Americans - eager to travel and see the world-still do not feel safe. This is evidenced in the hashtag communities that have formed around hashtags such as \#travelingwhileblack and \#sundowntowns. This paper shares early findings from an ongoing study of digital instances of Green's analog phenomena: risk and safety information sharing practices among the Black travel community in autonomous spaces. 


\section{Conceptualizing Autonomous and Liberated Spaces}

Autonomous or "safe" spaces were first conceptualized by social psychologist Kurt Lewin in his work on group dynamics and organizational change management. Lewin's work focused, in particular, on the problems of minority or disadvantaged groups in corporate spaces. Tasked with developing leadership training for high-level corporate executives, Lewin's work addressed two questions: What is it about the nature and characteristics of a particular group which causes it to respond as it does to the forces which impinge on it, and how can these forces be changed in order to elicit a more desirable form of behavior [11]? To address these questions, Lewin developed the concept of group dynamics. As Lewin's understanding of groups and group behavior expanded, he pioneered the practice of sensitivity training, recognizing that psychological safety was an imperative for honest communication - a prerequisite for organizational change. Lewin formalized his observations by creating group discussion settings wherein group members could provide honest feedback, disabuse others of harmful assumptions, and draw attention to implicit biases and other behaviors that were hindering effective leadership. Although the language of "safe spaces" was not used, because these group formations were intentionally designed around considerations of psychological safety, they can be considered among the first engineered safe spaces.

In her book "Mapping Gay L.A.," scholar and activist Moira Kenney traces the origins of "safe spaces" to gay and lesbian bars in the mid-1960s [10]. For the LGBTQ community, a safe space was a known place where - at least for an evening — one might be free of oppressive homophobic powers. Kenney dates the first use of the term "safe space" to the women's movement of the 1960s and 1970s, when safety equated to being free from oppressive patriarchal powers. Kinney writes that "safe space, in the women's movement, was a means rather than an end and not only a physical space but a space created by the coming together of women searching for community." In the Black community-where physical as well as psychological safety have historically been life and death concerns - churches, barbershops and hair salons, and historically Black colleges and universities (as well as many other Black-identified spaces) have all emerged as safe spaces. For Black Americans, safe spaces are those places where one can be free of the oppressive powers of whiteness and white supremacy.

Over time, the terms safe space and autonomous space have been used interchangeably in the United States to represent group spaces that have three primary attributes: a commitment to the physical and psychological safety of all members; a commitment to freedom from oppression; and a commitment to a common-often, but not always political-cause. Autonomous space thinking has also led to cultural shifts such as anti-oppression training, auxiliary spaces for identity groups to meet and organize, and gender-neutral bathrooms.

Some critics of safe spaces argue that autonomous spaces have become limited to ritualized relationships and codified behaviors. Others have argued that the creation safe spaces does not guarantee inclusion, particularly for those who exist at the intersections of identity groups. Black women, for example, might find that their safety challenged in Black spaces as women, and in women's spaces because of their blackness. In response to these critiques some have adopted the term safer spaces, indicating that a given autonomous space is safer than non-autonomous spaces. The most compelling argument for expanding autonomous space thinking, however, is that in addition to autonomous spaces, what is needed are new and inventive ways of fostering honest communication to effect change.

The liberated space, then, as I have conceptualized it, emerges from the ability to abstract and reconsider extant spaces-whether autonomous or not-to see the subversive qualities in their architecture and to recognize their creative and liberatory potential. While a safe space or autonomous space is a place of security and psychological safety, the liberated space takes the architecture of safe spaces and reimagines them as sites of liberation.

Social media communities like Facebook Groups are frequently designated as autonomous spaces for group members. Facebook Groups that are dedicated to the Black travel community, for example, typically require that members self-identify as Black (freedom 
from the oppressive powers of whiteness and white supremacy) and be interested in travel (common interest or cause). Moreover, these groups utilize language designating them as safe spaces: the travelers in these social media communities are seeking information that mimics The Green Book. Members of the Black travel community seek information from other Black travelers about where they can anticipate being welcomed, where they will be safe, where there is the potential for danger.

Through a detailed investigation of Black travelers in two distinct categories of social media communities (Facebook Groups and Twitter hashtag communities) I ask:

1. What are the similarities and differences between the utilization of Black Travel Movement social media communities and The Negro Motorists Green Book?

2. How do black travelers create liberated spaces in Black Travel Movement social media communities?

3. What are the affordances of social media communities that facilitate the movement from autonomous space to liberated space?

\section{Method and Design}

This study focuses on two distinct categories of social media communities-Facebook Groups and Twitter hashtag communities - to analyze their cultural and discursive construction. Data was collected from three anonymized Facebook Groups (BTM1, BTM2, and BTM3) and three primary hashtags - \#travelingwhileblack, \#greenbook, and \#sundowntown. Using methods adapted from Critical Technoculture Discourse Analysis-which draws on technology studies, communication studies, and critical race theory to understand how culture shapes technologies - and Grounded Theory, this study applies critical race and technoculture theories to information technology artifacts and their accompanying online conversations [3]. For this study, the Twitter and Facebook Group interfaces and platforms are as much a part of the data as the accompanying conversations. The data represented in this study was collected from January 2017 through early June 2018, representing nearly 18 months of longitudinal data. The data was then coded, conceptualized, and categorized in keeping with a Grounded Theory methodology. The larger project is a decade-long longitudinal study beginning in 2008 and aims to determine if and how autonomous social media community spaces become liberated spaces over time and what technical affordances may contribute to this shift. The lager project also seeks to determine whether certain social and cultural fears about travel can be mapped to political and/or historical moments - the 2016 United States presidential elections, for example. Of potential interest-but not significance - to this study is an early finding from the larger project that a particular temporal emphasis on \#travelingwhileblack posts has emerged. Centering around what might be called "travel seasons," the frequency of BTM activity increases during 3 two-month periods: Nov/Dec (Winter Holidays), May/June (Early Summer), and July/Aug (Late Summer). As such, much of the data for this study comes from these months.

BTM1 is a closed African American travel community Facebook Group with approximately 45,000 members. Group rules prevent using the group as a commercial space and mandates an environment free from bullying and hate speech-a safe space. The group hosts live events including chats with travel experts, and importantly, organizes group travel opportunities for community members, who must self-identify as African American or Black.

BTM2 is also a closed Facebook Group that specifically caters to the travel concerns of black women. Dedicated to strengthening the bonds of sisterhood through travel, BTM2 has about 22,000 members who self-identify as Black women. BTM2 was created to allow Black women to connect online and share their travel goals and dreams. Like BTM1, this social media community organizes group travel for community members. The moderators of BTM2 state explicitly that all posts to the community are reviewed before being posted and warn that community members in violation of anti-harassment and hate speech policies will be blocked and removed without warning or exception.

Like BTM1 and BTM2, BTM3 is a closed Facebook Group for self-identified Black travelers. With almost 350,000 members, BTM3 is a community of Black travelers who share an interest in cultivating new 
friendships and experiences through worldwide travel. BTM3 boasts professional travel agents in addition to shared community expertise. New membership is expressly encouraged in BTM3; in this way it differs from BTM1 and BTM2.

Unlike Facebook Groups, Twitter users have little to no expectation of privacy. While it is possible to create more private spaces on Twitter by locking accounts or limiting the audience for Tweets, this process is tedious and must be done on an account by account block basis. Twitter users form social media communities primarily through the use of hashtags. As evidenced by the phenomenon of Black Twitter, however, Twitter allows for insider/outsider knowledge to be shared using coded hashtags that people seek out based on individual identity characteristics. Similarly, combination hashtag searches can yield specific results for people who belong to a specific community. For example, a black motorist driving through Mississippi might search Twitter for the hashtags \#drivingwhileblack, \#travelingwhileblack, and/or \#sundowntowns in combination with \#Mississippi to seek information from other Twitter users about travel conditions for Black people in that state. For this study, three primary hashtags - \#travelingwhileblack, \#greenbook, and \#sundowntown-were used. Additional hashtags were also captured to account for spelling and ontological discrepancies (including \#travellingwhileblack, \#drivingwhileblack, \#thegreenbook, and \#sundowntowns). Combination terms, derivative terms (including those derived from \#safe, \#risk, and \#danger), and Boolean search were also used alongside the three primary terms to broaden search results and yield richer and more robust data. In total, twelve hashtags in five categories were used for this study, including over 100 original posts (OP) and close to 200 responses (RES) for a sample dataset roughly equivalent to 300 social media posts from the Black Travel Community (see Figure 1. Black Travel Movement [BTM] Frequently Used Words).

\section{Findings}

There are many more similarities than differences between how contemporary black travelers use Black Travel Movement social media communities and how mid-century African Americans used The Negro
Motorists Green Book, and the differences are mainly the result of technological affordances. One notable exception is that while The Green Book had pages of advertisements pointing travelers to auto repair shops, restaurants, and other Black-owned or Black-friendly businesses, all three BTM Facebook Groups expressly forbid advertisements in their community spaces. Despite this, because Facebook's profit model is based on advertising, users are inundated with ads as a regular part of the Facebook experience. The advertisements that Facebook users are likely to see may relate to travel, but will likely lack the nuance of addressing the specific needs of Black travelers. In the case of The Green Book, advertisements were intended to act both as a means of fiscal sustenance and as wayfinding tools to locate safe and dependable services. One finding from this study that Black travelers have used social media communities in a variety of other ways to seek trustworthy information: the information needs addressed by The Green Book are the same needs that continue to be expressed today by the Black Travel Movement.

As one example, BTM community members make use of Facebook's Recommendations feature to solicit information from fellow travelers. Facebook's Recommendations feature, which debuted in October 2016, allows users seek recommendations through their posts. Once the feature is enabled, Facebook Friends can comment on those posts with suggestions which Facebook then maps using GIS technology to allow the user to see a visual representation of Recommended places. Another similarity is the use of both The Green Book and social media communities to share legal information. While The Green Book included a state-by-state listing of state statutes on discrimination as they applied to "public accommodation or recreations" to aid travelers in having a "vacation without aggravation," many members of the BTM community use Facebook Groups and Twitter to inquire about current laws - both domestic and international-that would specifically impact Black travelers [7]. Black travelers in the social media communities studied here sought recommendations for everything from Black-owned businesses (around the globe) to patron and Black-owned hotels to places for Black women to have their hair styled or braided and places to find food prepared by Black chefs. 


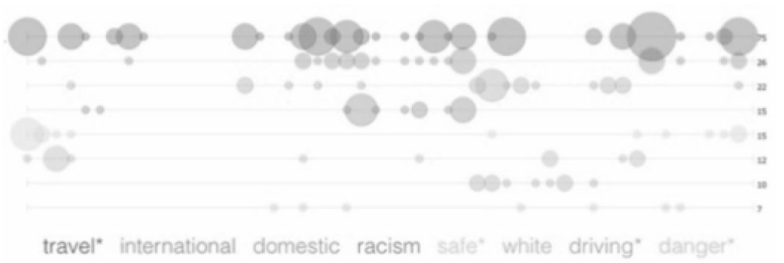

Figure 1. BTM Frequently Used Words

Points of interest, monuments, and markers represent another similarity between how African American travelers used The Green Book and the utilization of social media communities by contemporary Black travelers. The Green Book included points of interest in each state's listing, particularly New York-the Empire State Building, Coney Island, Prospect Park, the Apollo Theatre. In Black travel social media communities, travelers inquire about sightseeing around the globe - and many experienced travelers report that Black travelers are frequently the sight to be seen. A striking difference between The Green Book and BTM social media communities is that in Black travel social media communities a common theme is spectacle, or the feeling of being on display. WTBFs (well-traveled Black folks) frequently alert new Black travelers that ogling-particularly in predominantly white parts of the United States and in some Asian countries - and requests to be photographed (mostly in Asia) are common experiences. Although many embraced the curiosity displayed by those less familiar with darker hued skin, some felt they were being treated as if they were "animals in a zoo."

Finally, tips for safety and preparedness were common themes in Black travel social media communities just as they were central to the success of The Green Book. Information about travel visas, climate, currency exchange, and dress code are common to both resources. Of particular note is the explicit interest in Black travel social media communities in law enforcement and other travelers' experiences with and perceptions of racial bias among law enforcement officers. Many people recommended mobile applications for safe travel, including the Waze app, which allows for travelers to track where police cars are posted. The findings from this study suggests that when it comes to travel in the continental United States, travel by car is still as much of a concern as it was during the Jim Crow era when The Green Book was published. In response to an original poster's (OP) inquiry about driving across the country, common themes for safety were attention to time of day ("begin at 6 a.m., be in a hotel by 3 or 4 p.m." and "only daylight travel" were common warnings), an awareness of sundown towns, careful selection of travel routes (sticking to main thoroughfares, avoiding back roads or poorly lit roads), and traveling with a full tank of gas at all times.

As evidenced by the data in this study, Black travelers use social media communities in the service of safety and self-preservation. Black travel social media communities are themselves designed as safe spaces, allowing Black travelers to ask questions in an environment free from judgment and oppression as well as from potential violence and danger. How do Black travelers extend these safe-or autonomous - spaces to create the conditions of possibility for liberation, to be liberatory and liberated spaces?

The liberated space reflects the architecture of the existing space in new ways, reading it against the grain and using it to subvert oppressive powers. For Black travelers, the act of creating closed autonomous safe spaces within the broader global context of the Facebook platform is already a form of liberatory practice. This study suggests, however, that Black travelers have also begun to move toward liberated space thinking and practice in BTM social media communities. Entrepreneurship is one example of this shift toward liberated spaces. The Green Book was a service offered to African American travelers as an information resource. Although information sharing remains central to the functions of BTM social media communities, Black travelers have begun to use the affordances of social media to create services and information resources where they did not previously exist. Black travelers, aware of several racist incidents connected to a well-known accommodation marketplace created alternatives to the popular service. Responders to queries about the racist incidents direct the OP to these Black-owned or black-friendly alternatives (others also ask for more details about the city, the property, and the host so that they can avoid a similar situation). In addressing a need and a safety concern expressed in an autonomous space by creating new short-term rental 
services such as Innclusive, NoirBnB, and NoireBnB, Black travelers have created a liberated space: whereas this closed social media community was architected as a safe place to discuss concerns about how to avoid the racism of the popular short-term rental service, it is now a place of empowerment where Black travelers encourage one another to both create new solutions to the problem of \#travelingwhileblack and encourage support of fledgling Black businesses. This entrepreneurship that has emerged in response to claims regarding the popular service's permissiveness around racist hosts and guests is an unexpected but critical finding; it is also part and parcel of the BTM's observable liberatory praxis.

The ability to openly discuss - and disagree about-concerns about safety and the workings of oppressive powers in BTM social media community spaces is also noteworthy, particularly in the case of the Twitter hashtag community. A common theme expressed by Black travelers is the expectation that they will encounter racism in certain global regions. Posters noted experiencing more frequent racist incidents in Asia (particularly Japan) and Europe (particularly France and Germany). Similarly, concerns about of colorism frequently arise in asking about traveling to majority Black or ethnically diverse countries such as the Dominican Republic_-where dark-skinned Black people frequently experience colorism as a result of conflicts between the Dominican Republic and Haiti-and many African nations, where Black travelers from the United States are looked down upon because they are Americans. Warnings issued by the NAACP and AAA were also among the top content that was shared in Black travel social media communities, including the recent warning for Black travelers in Missouri and the travel warning for African Americans on American Airlines. What is notable about these discussions in the context of the Twitter hashtag community is the openness of the Twitter platform. Another unexpected but meaningful finding from this study is that Black travelers, using coded language and hashtags (used in addition to the hashtag terms that were used in conducting this study), communicate about global racism in open spaces, making their use of Twitter considerably less like a safe or autonomous space and more akin to liberated space. Black travelers on Twitter have found ways to create autonomous spaces with secret hashtags, using the affordances of the platform to their advantage and making from its architecture a place where secret, or "underground," conversations can happen in plain sight.

\section{Discussion and Conclusion}

The social media communities that I've been examining in my research on Black travelers and the Black Travel Movement are one example of many diverse identity- and inclusion-based social media communities on the internet. Part of what makes the Black travel social media communities unique, however, is that each one shares similar goals around opening and creating autonomous spaces for inquiry and discussion. Each social media community studied here also enacts everyday practices as radical experiments in spaces that are alternative to, within, and against oppressive powers, creating liberated spaces. The openness and other social features of the Twitter interface and platform permit "underground" discourse to occur in plain sight. The affordances of Facebook's advertising and group moderation models have encouraged entrepreneurship and the growth of Black-owned and operated businesses around the globe. While it is a matter of national shame that spaces that replicate the function of The Negro Motorists Green Book are still necessary in the United States as a result of injustice, indignity, and the perpetual threat of violence, it is a testament to the resilience of Black travelers that from the need to create autonomous spaces liberatory praxis has emerged. Black travelers have taken the architecture of internet interfaces and repurposed them into tools of liberation. While this ability to shift an autonomous space to a liberated space is not new in the Black community, it is always a revolutionary act to use the master's tools to dismantle the master's house.

What is equally compelling about the findings from this study is that although the parallels between the African American motorists of the 1940s, the contemporary Black travel community, and the Underground Railroad may not immediately apparent, they are striking. While escaping enslavement for Black people is in no way comparable to leisure travel, it can be argued that Black travelers in the United States have become 
adept at the art of safe passage throughout history by mastering the use of networks, both analog and digital. The Underground Railroad was a network of secret routes and safe houses established in the United States in the 1800s. Used by African American slaves to escape into free states and Canada, the Underground Railroad was so named because of the use of rail terminology for coded communication. The language of The Green Book is similarly coded, although such a reading of The Green Book is another study altogether. The Black Travel Movement Twitter hashtag community has also adopted coded language to remain under the radar of community outsiders.

Another parallel that can be drawn is the resourcefulness that safe passage continues to require. Enslaved persons traveling the Underground Railroad needed not only safe passage, but safe accommodation-which was often brought about through creative problem-solving. Similarly, safe passage and accommodation in the mid- $20^{\text {th }}$ century United States is a concern that is at the heart of The Green Book. Today, Black travelers in the BTM Facebook Groups, having identified a pressing need for safe accommodation as they travel, have created new housing businesses and short-term accommodation networks. Recognizing the ongoing need for safe passage, Black travelers share tips on Visas and avoiding potentially deadly encounters with law enforcement. One notable difference, however, is modes of communicating about safe passage and safe accommodation; the Underground Railroad relied heavily on word-of-mouth. Oral instructions were given because the printed word was subject to discovery, writing technologies were the purview of white people, and enslaved persons were not permitted to be literate.

Finally, in a discussion of liberated spaces, I must address the common goals of freedom and liberation. The ultimate need for the Underground Railroad was to help enslaved people enter a place where they could be free. So, too, did Victor Hugo Green seek to offer Black travelers an experience free of worry and an opportunity to engage the world as a means of liberation and knowledge expansion. By embracing the methods of generations of Black travelers, the contemporary Black Travel Movement has created autonomous spaces, anointed safe spaces, and then transformed and revolutionized those spaces into liberated spaces that subvert oppressive powers and help Black people "get free."

As I continue to carry out work on what is an important and understudied topic, I expect to find more examples of Black social media communities adapting and subverting social media platforms and interfaces - as well as other digital media tools - in the ongoing struggle for liberation. Early examples of this kind of work such as Miller and Slater's 2003 study on internet use in Trinidad and Tobago - albeit with different methods, tools, and research goals - indicate that deeper and broader studies of tool manipulation and subversion to meet unique identity-group and community goals are needed [17]. Learning how different groups change or alter the intended purposes of internet-based and other digital tools to improve their quality of life has much to offer researchers in all disciplines.

Directions for future research include a broader investigation of some of the expected, unexpected, and most compelling findings of this study: entrepreneurship as a feature of liberated spaces; uncovering new affordances of old technologies; and investigating new understandings of technology from investigating their historical equivalents. Additionally, because this study specifically addresses Black- and African American-identified communities as part of the Black Travel Movement - and there is no extant body of literature that would extend this study beyond these communities - two additional avenues for potentially impactful future research are a broader diasporic or multiethnic study of online autonomous spaces that have seized upon liberatory praxis, and studies that consider the liberatory affordances of social media in autonomous online spaces created by other (non-Black) marginalized communities.

\section{References}

[1] Bonilla, Y. and Rosa, J. “\#Ferguson: Digital Protest Hashtag Ethnography and the Racial Politics of Social Media," American Ethnologist, 2015, pp. 4-17.

[2] Breuer, A. Landman, T and Farquhar, D. "Social Media and Protest Mobilization: Evidence from the Tunisian Revolution," Democratization, 2015, pp. 764-792. 
[3] Brock, A., "Critical Technocultural Discourse Analysis," New Media and Society, 2016, pp. 1085-1103.

[4] Brock, A., "From the Blackhand Side: Twitter as a Cultural Conversation," Journal of Broadcasting \& Electronic Media, 2012, pp. 529-549.

[5] Florini, S., “Tweets, Tweeps, and Signifyin': Communication and Cultural Performance on 'Black Twitter,"' Television \& New Media, 2013, pp. 223-237.

[6] Franz, K., "African-Americans Take to the Open Road," in Franz, K. and Smulyan, S., Major Problems in American Popular Culture, Cengage Learning, 2011.

[7] Green, V.H., The Negro Travelers' Green Book, Victor H. Green and Company, New York, 1963-1964. See: Schomburg Center for Research in Black Culture, Manuscripts, Archives and Rare Books Division, The New York Public Library. "The Negro Travelers' Green Book: 1963," New York Public Library Digital Collections.

[8] Jackson, S. and Foucault Welles, B. "Hijacking \#myNYPD: Social Media Dissent and Networked Counterpublics," Journal of Communication, 2015, 932-952.

[9] Jackson, SJ. “(Re)Imagining Intersectional Democracy from Black Feminism to Hashtag Activism," Women's Studies in Communication, 2016, pp. 375-379.

[10] Kenney, M., Mapping Gay L.A.: The Intersection of Place and Politics, Temple University Press, Philadelphia, 2001.

[11] Kippenberger, T., "Planned Change: Kurt Lewin's Legacy," The Antidote, 1998, pp. 10-12.

[12] Knight Steele, C., "Black Bloggers and Their Varied Publics: The Everyday Politics of Black Discourse Online," Television and New Media, 2017, pp. 112-127.

[13] Knight Steele, C., "The Digital Barbershop: Blogs and Online Oral Culture Within the African American Community," Social Media and Society, 2016 pp. 1-10.

[14] Korn, J. "Black Nerds, Asian Activists, and Caucasian Dogs: Online Race-based Cultural Group Identities within Facebook Groups," International Journal of Interactive Communication Systems and Technologies, 2015, pp. 14-25.

[15] Kuo, R. "Racial Justice Activist Hashtags: Counterpublics and Discourse Circulation," New Media and Society, 2016, 495-514.
[16] Lewin, K., "When Facing Danger," in Lewin, G. W. (Ed.), Resolving Social Conflict, Harper \& Row, London, 1939.

[17] Miller, D. and Slater, D., The Internet: An Ethnographic Approach, Berg, Oxford, 2003.

[18] Nakamura, L. "I Will do Everything that Am Asked': Scambaiting, Digital Show-Space, and the Racial Violence of Social Media," journal of visual culture, 2013, pp. $257-274$.

[19] Noble, S. "Teaching Trayvon Race, Media, and the Politics of Spectacle.” The Black Scholar, 2014, pp. 12-29.

[20] Sharma, S. "Black Twitter? Racial Hashtags, Networks and Contagion," New Formations, 2013, pp. 46-64.

[21] Sutherland, T. "Archival Amnesty: In Search of Black American Transitional and Restorative Justice," Journal of Critical Library and Information Studies, 2017.

[22] Tufekci, Z. and Wilson, C. "Social Media and the Decision to Participate in Political Protest: Observations From Tahrir Square," Journal of Communication, 2012, pp. 363-379

[23] Williams, J. A., This is My Country Too, New American Books, New York, 1965. 